436 Berwendung bes Dynamits zur Stoutrobung und Bobenvorbereitung.

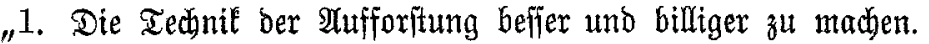

2. Die beftehenden $\mathfrak{B a ̈ l b e r ~ z u ~ v e r b e f f e r n , ~ i n t ~ e i n z e l n e n : ~}$

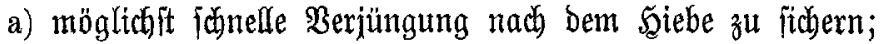

b) bie majfe $=$ und geldertraggreichiten Syolzarten za fultivieren;

c) Die Borräte geringer Wibaldungen žu heben;

d) Dualität uno Form ber Bäume aufs befte zut ftetgern;

e) ben gröpten zettlich möglicten Maffenzumacts zu erzielen bet befierem Salluffe und befferer Dualität Der Maldungen."

Dieje Ziele find wohl erreidbar. Und ferner ftellte biejer Bericht ober Inntrag als das \$rinzip jeder forftlithen \$olitif den Sah auf:

„Ein $\mathfrak{B a l b}$ muß́s als ein probuftives Sapital, unterworfen einer

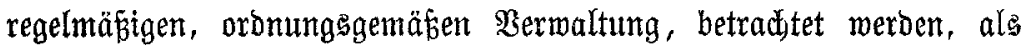

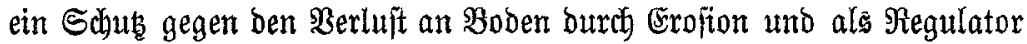
Der Birtulation bes $\mathfrak{B a f f e r s . "}$

Dem Seerrn $\mathfrak{B e r f a f f e r ~ m o ̈ g e n ~ b e i ~ \Im 4 t h a f f i n g ~ j e i n e s ~ A n t r a g e s ~ b i e ~ o ̈ b e n ~}$ Steinwüiten nidft mux jetnes engeren Baterlandes als aud bie bes Rarftes und bes Srientes vorgeidgwebt haber.

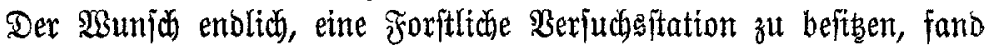

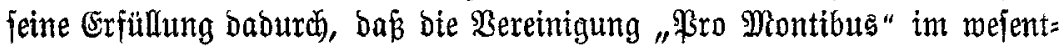
licten bieje 2 ufgabe übernommen hat.

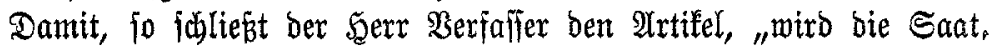

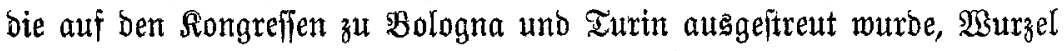
faffen und zut Entfaltung lommen, žum Woble von ganz Ștalien, Deffen

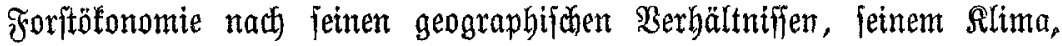
feinen Şandelsbeziehungen und jeiner Snduftrieentwitłlung ben britten Teil ber ganzen nationalen B̈tonomie autsmadhen follte". -rto-

\title{
Zlus Zlmerifa.
}

Bermenoung bes Dynamits zur Stodirobung und Boben= vorberettung.

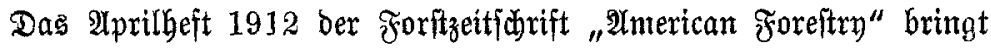
einen $\mathfrak{A r t i f e l}$ über bie $\mathfrak{A}$ nwenbung bes Dynamits zur Stodrobung uno Bobenvorbereitung zur Baumpflan\}ung.

Die brei gemöhnlich in 2 Unwendung ftehenden Methoden ber Stoce robung find: 1. Şerausziehen ber Stö̌e mit Majchinen, 2. Entfernung burb Feuer, 3. Serausfohteß̃en mit Dnnamit. Şede bat unter beftimmten

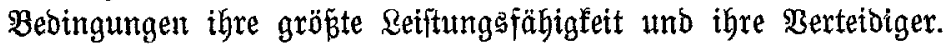

Die 2 nnmenbung bes Dynamits hat fid nad neueren Erfahrungen 
Berwenoung bes Donamits zur Stoftrodung unt Bobenborbereitung. 437

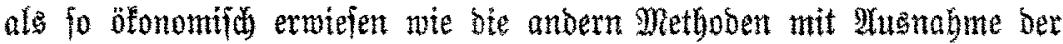
Robung fleinerer Stöde.

Die Wumendung unb bie Binfung bes Dynamits ifi befannt. Was aber sin Borteil biejer neuen Methooe gu feitt foheint, if, baj burd bie Explofion ber \&abung, welde ben Stod herausmint, aut ber Untergrumb zertrümment wirb. Die Wirhng Giernon it, baj beim 3uridfallen ber Geraugefolettoerten Exbmaffen in bie Eprengöfinung fid ber von inten nad oben gertffene näbrftoffretde Boben mit bem Dberflähenboben mengt,

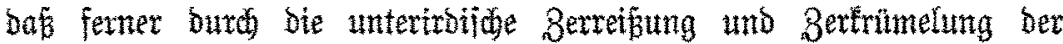
Boben in einen 3 uftano verjest wirb, weldet ben Atmoiphärilten einen leidten 3 ugang bis in gröbere Titefen, eine leidotere Bearbeitung bes

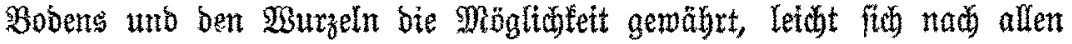
Seiten aubbreiten zu lömnen.

So zetgten aud bie crfabrungen auf Wetzenfelbern wie in Dbft= gärten, wo man fich biefe Beobathtungen zunube madte und jebst vielerorts

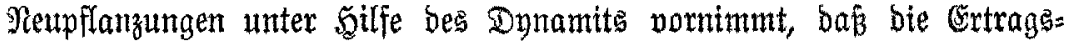
fäbigfeit günftiger als bet anoern Methoden beeinfluf̧t war.

(s) haben fich als Ergebmifie biejes neuen Serfahrens nach ben Beobachtungen ber lebsten Sabre namentlid in Gartengrunbftüuten folgende Birfungen feftitellen laffen:

Erftens: Bobenvorbereitung burch Dynamit ichaltet ben Berluft junger Fffanzen wäbrenb bes eriten Sabres aus.

3wettens: Das Pflanzgefdäft vollziteht fich raider als unter ben alten Methoben besjelben.

Drittens: Bäume, bie "mit Dymamt gepflantzt" wutron, fommen ein ober zwei sabre früher zum Tragen als bie auf bie gewöhnlide 9 Irt gepflanzten. Shierzu wie zu ben übrigen Beobađitungen fitto bem Irrtifel abbilbungen nach \$hotographien beigegeben, welde bas $\mathfrak{B o r}=$ getragene bettätigen.

Sierten: Derartig behanbelte Bätme zeigen ein fonnelleres Madjutum

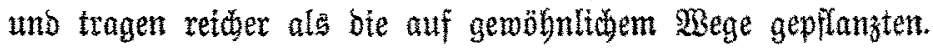

Diteje Säbe werben nit Erjabrungen grober Gartenbetitber uno

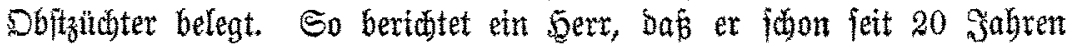
zux Pflanzutg Feiner Bäume bas Dynamit vernenbe unto baj er hter= burdh gegenuber feiner friberen ant unb Weife zwet Sabre unter jechs profitiere, $\mathfrak{D}$. $\mathfrak{h}$. bei einem mit Dynamit gepilantzten Baume erzielt $\mathfrak{e r}$ it

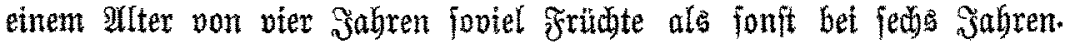

Die oben fobon angeführten Gtünbe, bie Durdiluftung bes Bobent,

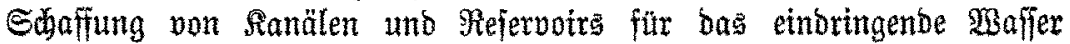
unb tiefere Berfiümefung bes $\mathfrak{B}_{0}$ bens für Beföroerung einer energifden 


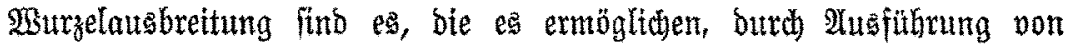
Sprengungen (wobl aber boh nur ibwache sabungen) zwifichen ben Reiben alter ober im Ertrage nidjt genügenber Dbitbäume bieje zu neuem Badestum, neuer Energie anzuregen.

-rto-

\section{Unterrichtsfurje für praftijahe forftwirte}

\section{an der R. .. Gochichule für Bodenfultur in Woien 1912.}

Bei ber XII. Sauptwerjammlung bes Deutichen Forftuereing zu Sönigaberg im Sabre 1912 itand als widtigites Themta auf ber Tages: oroming "Die Fortbildung bes Foritverwaltungesperionales". Inter ben mancherlei Mitteln zu beren Förberung murben aud bie Fortfilbungs:

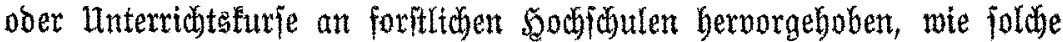
in Deutfoland lebiglid zweimal an ber Foritafabemie Eberewalde burch bie bortigen afabemifident Refrex abgebalten wutroen, im Sabre 1896

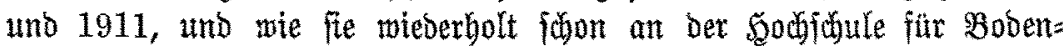

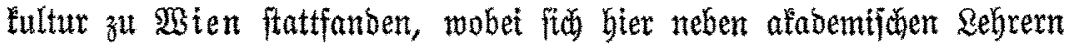
autif etzzelne bervorragende Månter ber \$rarts beteiligten.

uैber ben Fortbildungsturjus ză Eberswalbe im Sabre 1911 hat

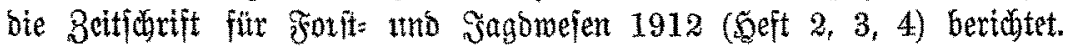
Sn Mien fand ein folder "Interridjtsfurjus" im Mai bes heurigen sabres ftatt, und es bürfte unjern sejern bas Frogramm biefes Surjes

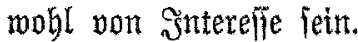

Montag, ben 20. Mai.

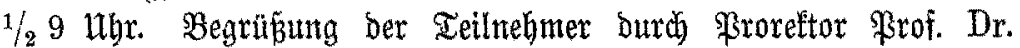
Cieslar in Sertretung Sr. Magnifizenz bes Reltores.

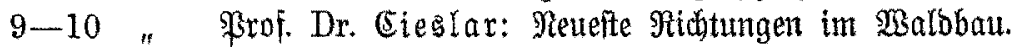

10-11 "Dozent Sofrat Sdiffel: Utber bie Fehler ber Snhalts: ermittelung von Rangholz nach ber Mittenftänte; Dar: ftellung anberer praftifiter fubterungsintethoden.

3-4 " Sofrat \$rof. Dr. von Shullern zu હdrattenbofen: utber foritwirtichaftliche Genofienfibaten.

4-5 " Dr. Babrmunb Riegler: Die wiötigften Erfahrungen uno Forichungsergebniffe auf Dem Gebiet ber Sagbfunbe.

Dienstag, ben 21. Mai.

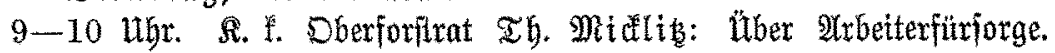

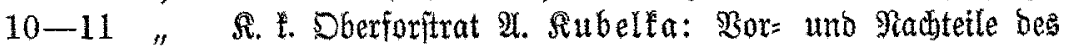

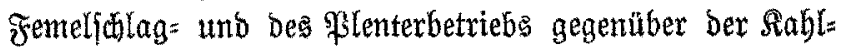

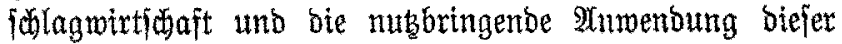
Betriebsartent in ber \$raris. 